\title{
Introduction: Mental Health among Marginalized Communities
}

\author{
Special Issue Editors
}

Tabbye Chavous, director of the National Center for Institutional Diversity; associate dean for Diversity, Equity, and Inclusion in the College of Literature, Science, and the Arts; and professor of education and psychology at the University of Michigan.

Sherry Molock, senior research fellow at the Steve Fund and associate professor of clinical psychology at George Washington University.

Laura Sánchez-Parkinson, manager of public scholarship initiatives at the National Center for Institutional Diversity at the University of Michigan and former director at the Steve Fund.

The National Center for Institutional Diversity (NCID) at the University of Michigan and the Steve Fund, a national nonprofit organization dedicated to the mental health of young people of color, launched a partnership in 2019 to raise awareness of mental health among students of color and offer evidence-based interventions and best practices for educators and decision makers. We did not know that mental health and emotional well-being would become even more critical for marginalized communities as we entered into the COVID-19 pandemic and nationally experienced a myriad of discriminatory and violent 
incidents against marginalized communities. As our nation continues to grapple with this trauma, we have once again partnered to release a special issue on mental health among marginalized communities.

The articles in this special issue offer insights into the diverse mental health experiences from marginalized communities and examines how we can advance culturally relevant and equitable policies and practices to support their mental health and emotional well-being.

Scholars in this special issue contextualized the social identities (i.e., race, gender, sexual orientation, age, etc.) and the intersecting identities of the communities they studied and examined the inequalities in access and utilization of mental health services. For instance, Miller et al. explored the mental health of LGBTQ+ college students with disabilities and the complexity in the relationship between mental health and disability diagnosis and outcomes. They interviewed twenty students, providing insight into the benefits of counseling services but also how these services often fell short in offering culturally relevant resources and services.

Leath and Jones's mixed-methods study also examined college students-specifically Black students at two diverse institutions-and explored the impact of racial climate on mental health service utilization. They found that students who perceived the campus as more welcoming to racial and ethnic minority students reported higher positive mental health. However, Black college students reported facing barriers to counseling services when they would realize there was inadequate representation of Black counselors and counselors who they could meaningfully connect with.

McCallum et al. studied Black doctoral students, collecting data during the COVID-19 pandemic and offering timely insights. The role of a doctoral student often comes with added academic stressors and longer-term relationships with their faculty advisors and the institution. As instructors, they also carry an additional role of supporting undergraduate students. Before the pandemic, doctoral students reported ongoing unsuitable and irrelevant mental health services for graduate students and pressure to keep their mental health chal-lenges to themselves for fear of repercussions from faculty. While the 19 pandemic brought its own challenges, particularly imp- 
act their productivity, doctoral students shared an increased focus on their mental health and a growing attention to understanding the impact of racialized trauma on themselves and their communities after the killings of a number of unarmed Black people during this same time period.

Yazdiha and Boen homed in on the immediate and long-term effects and the collateral consequences of racialized police violence. Using longitudinal data (text from over two hundred thousand unique Twitter users) and two national cases-the killings of Michael Brown and Tamir Rice-they evaluated the emotional and psychological effects of police violence to identify and trace trauma. Their findings suggest that the tweets became more emotionally negative after both of the shootings, particularly for Black Twitter users, which has nega-tive consequences to their well-being.

Brown has examined the unexplained phenomenon that although Black older Americans have experienced more stress exposure and discrimination, they have exhibited lower rates of depression and anxi-ety disorders relative to white older Americans. In this study, drawing from national data on adults aged fifty-one and older, Brown argues that because Black Americans have experienced more chronic stress-ors over their lifetime, they have developed stronger coping mecha-nisms and have reframed situations to reduce the stressfulness of an experience. In addition, Brown also highlights the need to revisit the measures of stress that may not be capturing the unique experiences of Black Americans.

Zhang and Grant also analyzed national data, focusing on examin-ing three different waves of data from childhood to adulthood. Exam-ining across racial groups (Black, Latinx, Asian, and white) they find that parent-child relationships-particularly maternal closeness-and parent-living arrangements are significant predictors of depressive symptoms as children age into adulthood. Non-Latinx Asian respon-dents in particular demonstrate significant lower depressive symptoms compared to non-Latinx white respondents when examining their maternal closeness and parent-living arrangements. 
Acknowledging the diverse experiences and intersecting social identities of a person is critical in order to develop policies and practices that will support the mental health and emotional well-being of people from marginalized communities. For relevant programs and interventions, we must pay attention to the unique needs of a population. Delmonaco et al. explored the needs of LGBTQ+ youth to specifically address how youth can leverage online resources to access health-education information. By hosting three focus groups, Delmonaco and Haimson identified key themes for what LGBTQ+ youth need, including how to access mental health services as a minor and how to care for their mental health while in toxic environments that they cannot escape. Following the focus groups, researchers also held design sessions for youth participants to develop an LGBTQ+ health web resource. Through the project, researchers are also partnering with the Community Health Access Initiative to launch an evidencebased resource website for LGBTQ+ youth.

Ball and Skrzypek offer another promising intervention, a unique program that links school transformative social-emotional learning and intergroup dialogue, two well-informed approaches to engag-ing students in becoming more self-aware and developing relational skills. The program engaged students across six predominantly white high schools in a one-day peer-to-peer learning and dialogue experience. Students reported feeling emotionally safe in the space and an increased sense of belonging, particularly for students who felt isolated at their predominantly white school. Students' increased selfawareness and empathy for others were critical findings, which are relevant to understanding youth's well-being.

The collection of pieces presented in this special issue give us insight into the unique mental health experiences and needs across marginalized communities, and intersecting identities must be taken into account when analyzing a population and developing interventions. The diverse methodological approaches presented also give light to the varied ways in which we can further our understanding of complexities across groups. In this process, authors noted challenges and gaps that remain in research and practice, particularly in how we 
continue to measure mental health and develop interventions that are culturally relevant.

As we continue to understand the number of impact areas that the COVID-19 pandemic has brought to our communities, monitoring the mental health of marginalized communities must continue to be critically important. As a nation we have also continued to increase awareness of the mental health effects that come from racism, sexism, xenophobia, homophobia, and other forms of oppression, which are unique to each community but have long-term implications for the health and success of all communities. It is important and necessary for scholars to continue to contextualize and inform these phenomena while offering practical implications that can be applied across sectors and our society. 